\title{
THE DISTRIBUTION OF INTERSTELLAR MOLECULES IN DUST CLOUDS AS DERIVED FROM LABORATORY EXPERIMENTS
}

\author{
H. D. BREUER
}

Institut für Physikalische Chemie II, Universität des Saarlandes, Saarbrücken, W. Germany

\begin{abstract}
Irradiation of adsorbed simple gases leads to the formation of rather complex molecules. On the basis of the wavelength dependence and the cross sections for formation and desorption an average molecule density can be estimated. The wavelength dependence further shows that molecules can be formed in denser regions where they are protected from dissociating radiation. A simple model is proposed which shows the molecule distribution as a function of dust density.
\end{abstract}

\section{Introduction}

In the last few years several mechanisms have been proposed for the formation of interstellar molecules. For some of the diatomic radicals and molecules radiative association and charge exchange reactions in the gas phase seem to be mechanisms which at least in special regions of the interstellar space give satisfactory results (Solomon and Klemperer, 1973). The formation of more complex molecules consisting of four or more atoms can for several reasons not occur in the gas phase. Dust grains are involved and the reactions seem to be catalytical and possibly photo-catalytical. The purpose of this paper is to apply some laboratory results for molecule formation in photoinduced reactions at solid surfaces on the possibilities of molecule formation in interstellar dust clouds. From the wave length dependence of the formation process and of the destruction of molecules by photo dissociation a qualitative model of molecule distribution in dust clouds can be deduced.

\section{Experimental}

The experimental set up we used for studying molecule formation in photoreactions at solid surfaces has been described in detail elsewhere (Breuer, 1969; Moesta et al., 1969). Two different experiments were performed. In one experiment we investigated the wave length dependence of these photoreactions; in the other experiment the photo products were identified by a mass spectrometer.

The surface used in theses experiments were either metal films or metal foils, the metals being nickel, tungsten or rhenium. The gases introduced in the reaction system were $\mathrm{H}_{2},\left(\mathrm{D}_{2}\right), \mathrm{N}_{2} \mathrm{O}_{2}, \mathrm{CO}, \mathrm{NH}_{3}, \mathrm{CH}_{4},\left(\mathrm{CD}_{4}\right)$.

These gases were adsorbed in various combinations and ratios. The adsorption systems were irradiated either by an exploding wire light source (Moesta and Breuer, 1965) or by resonance lamps. The experiments were performed at room temperature.

Measuring the wave length dependence we found the most effective spectral region 


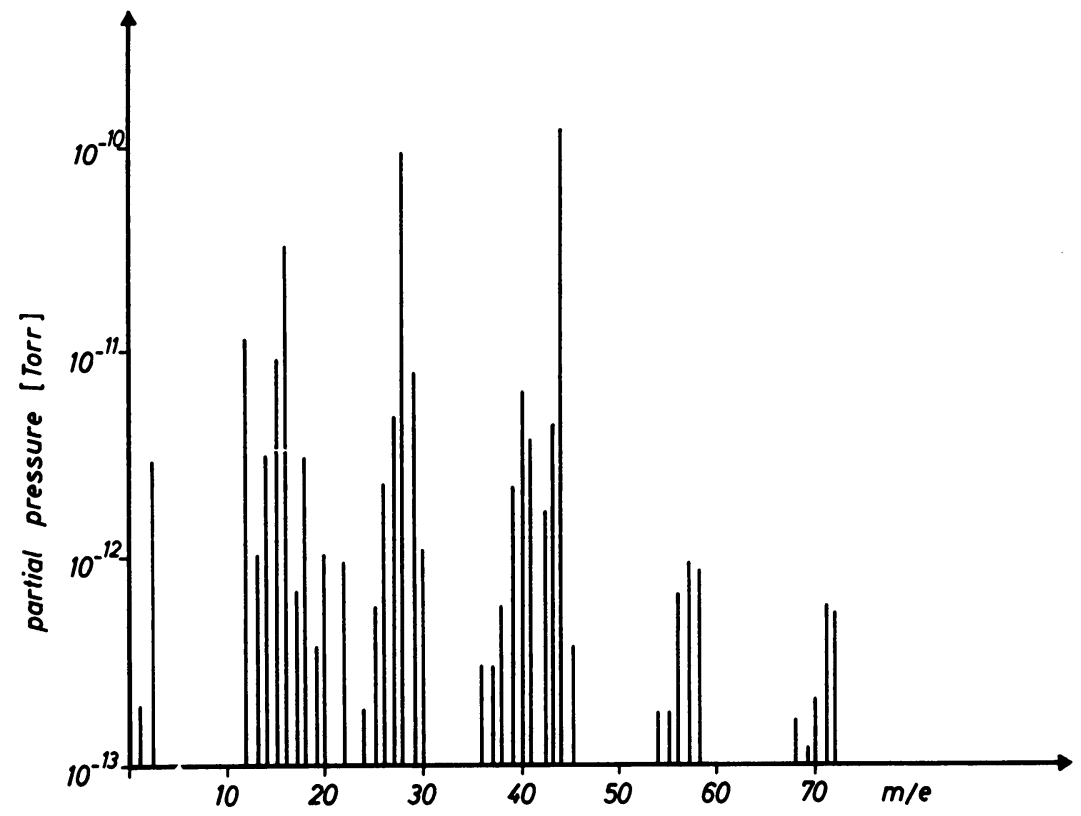

Fig. 1.

for changes in the chemical composition of the adsorption layer for all adsorption systems being between $2000 \AA$ and $3000 \AA$.

As an example for the photo production of molecules Figure 1 shows the mass spectrum which is obtained by irradiating a mixture of $\mathrm{CO}$ and $\mathrm{CH}_{4}$ adsorbed on tungsten. In this spectrum the peaks which are observed without irradiation are subtracted and only the photo peaks are shown. An analysis of this spectrum shows fragmentation peaks which are typical for the presence of aldehydes. Using $\mathrm{CD}_{4}$ instead of $\mathrm{CH}_{4}$ formaldehyde could be identified. Other photo products are hydrocarbon radicals and $\mathrm{CO}_{2}, \mathrm{C}_{2} \mathrm{O}, \mathrm{C}_{2} \mathrm{O}_{2}$ and $\mathrm{C}_{3} \mathrm{O}_{2}$. Irradiating adsorption layers containing nitrogen we could identify the products listed in Table $\mathrm{I}$.

For some molecules we were able to measure the cross sections for formation and desorption. These cross sections are shown in Table II.

\section{Discussion}

The experimental results show that by irradiating simple gases adsorbed at the surface of a solid rather complex organic molecules can be formed. The cross sections for formation and desorption indicate that photocatalytic reactions are a very operative mechanism for molecule formation. The formation of the observed molecules can only be understood if preadsorbed diatomic molecules, e.g. $\mathrm{CO}$, are dissociated by the radiation. By the interaction of the adsorbed molecule with the surface $\mathrm{CO}$ can be electronically excited with $\lambda \sim 2000 \AA$. From this excited state it dissociates to $\mathrm{C}$ and 
TABLE I

\begin{tabular}{ll}
\multicolumn{2}{c}{ Molecular weights } \\
\hline$m / e$ & Molecule \\
\hline 26 & $\mathrm{CN}$ \\
27 & $\mathrm{HCN}$ \\
29 & $\mathrm{H}_{2} \mathrm{H}$ \\
30 & $\mathrm{NO}, \mathrm{N}_{2} \mathrm{H}_{2}$ \\
38 & $\mathrm{C}_{2} \mathrm{~N}$ \\
39 & $\mathrm{C}_{2} \mathrm{HN}$ \\
40 & $\mathrm{C}_{2} \mathrm{H}_{2} \mathrm{~N}$ \\
41 & $\mathrm{CH}_{3} \mathrm{CN}$ \\
43 & $\mathrm{HNCO}_{4}$ \\
44 & $\mathrm{~N}_{2} \mathrm{O}$ \\
46 & $\mathrm{NO}_{2}$ \\
47 & $\mathrm{HCONH}_{2}$ \\
51 & $\mathrm{HC}_{3} \mathrm{~N}$ \\
\hline &
\end{tabular}

TABLE II

Cross sections for formation and desorption of molecules

$$
\begin{aligned}
& \sigma_{\mathrm{CN}}=1.5 \times 10^{-18}\left[\mathrm{~cm}^{2}\right] \\
& \sigma_{\mathrm{HCN}}=5 \times 10^{-19}\left[\mathrm{~cm}^{2}\right] \\
& \sigma_{\mathrm{H}_{2} \mathrm{CO}}=1 \times 10^{-16}\left[\mathrm{~cm}^{2}\right] \\
& \sigma_{\mathrm{CH}_{3} \mathrm{CN}}=1.5 \times 10^{-18}\left[\mathrm{~cm}^{2}\right] \\
& \sigma_{\mathrm{HNCO}^{2}}=1 \times 10^{-18}\left[\mathrm{~cm}^{2}\right] \\
& \sigma_{\mathrm{HCONH}_{2}}=1 \times 10^{-18}\left[\mathrm{~cm}^{2}\right] \\
& \sigma_{\mathrm{HC}_{3} \mathrm{~N}}=5 \times 10^{-19}\left[\mathrm{~cm}^{2}\right]
\end{aligned}
$$

$\mathrm{O}$ (Breuer, 1969). These atoms can react with neighboring $\mathrm{H}$-atoms or already formed $\mathrm{CH}, \mathrm{CH}_{2}$ etc. to form the observed molecules.

Applying these laboratory results to the conditions in interstellar space seems to be risky since interstellar dust grains are certainly not made of pure nickel or tungsten. However, experiments using soil or pulverized vycor as substrates show that very similar processes occur on non-metal surfaces as well. The major result of our experiments is that all the observed photoreactions occur in the spectral region between $2000 \AA$ and $3000 \AA$ a region which is almost inoperative in normal photochemistry in the gas phase since all of the gases relevant for astrochemical purposes are transparent in this spectral region. The interstellar extinction curve, however, shows that this radiation can penetrate into the clouds at a higher rate than the radiation below $2000 \AA$. Since for most of the molecules dissociation starts at about $2000 \AA$ appreciable life times can only be achieved if the molecules are shielded from the dissociating radiation by interstellar dust (Stief, 1971).

Calculations on the distances that molecules can cover during their life time show that they can only be formed in the regions where they are observed, i.e. in the dust clouds. If photocatalytical reactions are one of the processes responsable for molecule 
formation in interstellar space the conclusion which can be drawn from the experimental results should be at least in qualitative agreement with this fact.

The calculation of the molecular densities should in principle be possible by combining the experimental results with the calculated life times. The rate, $\boldsymbol{R}_{\boldsymbol{i}}$, at which molecules are formed and desorbed from the surface of a dust grain, is given by

$$
R_{i}=N_{\mathrm{ads}} \theta A \Sigma_{\mathrm{ph}} \sigma_{i}\left[\mathrm{~s}^{-1}\right],
$$

where $N_{\text {ads }}$ is the number of available adsorption sites per $\mathrm{cm}^{2}, \theta$ is the degree of coverage and is assumed to be unity in the following calculations. $A$ is the area of the dust grain, $\Sigma_{\mathrm{ph}}$ is the photon flux $\mathrm{cm}^{-2} \mathrm{~s}^{-1}$, and $\sigma_{i}$ is the cross section for formation and desorption. Since the chemical composition of the adsorption layer is one of the factors which determine the way in which the reactions proceed, $N_{\text {ads }}$ has to be divided up into the various gaseous constituents which can be involved in the reactions at the surface. The rate $r_{k}$ at which $k$-atoms of $k$-molecules hit the surface and stick to it is given by

$$
r_{k}=S_{k} u_{k} v_{n} A\left[\mathrm{~s}^{-1}\right] \text {. }
$$

$S_{k}$ is the sticking coefficient which for all atoms and small molecules in $\mathrm{H}$ I-regions is equal to or very close to unity, $n_{k}$ is the number density of the species $k$ and $v_{k}$ the corresponding velocity. Using the abundances relative to hydrogen and putting $v_{k} / v_{\mathrm{H}}$ where $v_{\mathrm{H}}$ is the velocity of a hydrogen atom the composition of the adsorption layer can be estimated. However, if reactions proceed in the surface layer this composition can be changed in an unforseeable way since newly formed radicals and molecules can occupy a part of the surface sites. The number density of molecules produced by this reaction may be obtained by multiplying Equation (1) by $\tau_{i}$, the life time of the molecule $i$ and the number density of the dust grains.

Applying this equation to the conditions in interstellar space, one can assume an adsorption layer consisting of $\sim 10^{14}$ 'reactive particles' and a mean cross section of $10^{-18} \mathrm{~cm}^{2}$. The other assumptions are: hydrogen density $n_{\mathrm{H}}=100 \mathrm{~cm}^{-3}$, dust density $n_{d}=10^{-10} \mathrm{~cm}^{-3}$, grain diameter $d_{g}=0.1 \mu$, grain surface $A=3 \times 10^{-10} \mathrm{~cm}^{2}$, radiation density $U_{\lambda}=4 \times 10^{-17} \mathrm{erg} \mathrm{cm}^{-3} \AA^{-1}$ The photon flux in the spectral region between $2000 \AA$ and $3000 \AA$ is then $1.5 \times 10^{8} \mathrm{~cm}^{-2} \mathrm{~s}^{-1}$. With an absorption in the visual of $A_{\mathrm{v}}=3$ the mean attenuation of the radiation is $10^{-3}$ and the corresponding mean life time is $10^{13} \mathrm{~s}$ (Stief, 1971). Inserting these numbers in the equation we obtain an integral molecular density of $4.5 \times 10^{-6} \mathrm{~cm}^{-3}$. This density has to be divided up into the different species according to their relative cross sections and life time.

As mentioned above the formation and the destruction of the molecules occur in different wave length regions. Photodissociation occurs in less protected regions mainly by photons having an energy of $6 \mathrm{eV}$ or more, whereas the formation reactions need only photons in the 4 to $6 \mathrm{eV}$-region. Since both the formation and the destruction of the molecules are proportional respectively inversely proportional to the number density of the dust grains the following model is postulated for the distribution of molecules in dust clouds. Figure 2 shows the spatial distribution of a dust cloud. This distribution has not necessarily to be Gaussian as drawn in the figure. Due to the 


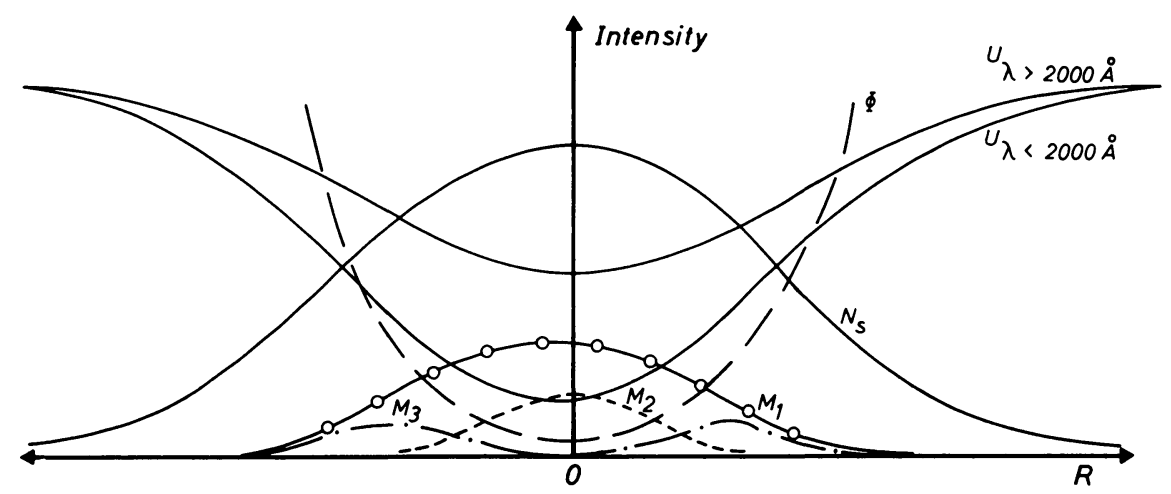

Fig. 2.

above mentioned proportionalities the model is valid for any shape of the cloud. The separation of the radiation in $\lambda>2000 \AA$ and $\lambda<2000 \AA$ is arbitrary. It shall only reflect the influence of the extinction curve on the radiation field. Since molecule formation is directly proportional to the dust concentration the highest molecule densitites can be expected in the center of the cloud. This is the case for the molecules $M_{1}$ and $M_{2}$. Having the same wave length dependence a higher concentration is obtained if $\sigma_{1}>\sigma_{2}$. If photons of higher energies are necessary to form a molecule this species will be formed in a region of the cloud where the grain density permits the radiation to penetrate with sufficient intensity. Here the formation rate will be lower since in the outer shell of the cloud the grain density is lower. The formation in an outer shell of the cloud is shown for the molecule $M_{3}$. The spatial distribution of each molecular cloud is limited by the dissociation in the interstellar radiation field. The curve indicated by $\Phi$ is the integrated quantum yield for the photo dissociation of the molecules. $\Phi$ is unity in dust free regions and decreases as the molecules are protected from the dissociating radiation by the interstellar dust.

In this simple model the molecules $M_{1}$ and $M_{2}$ form concentric spheres and are mixed in the core of the cloud while in an outer shell $M_{1}$ and $M_{3}$ are mixed. Any irregular shape of the dust cloud will be reflected in the distribution of the molecules and $M_{1}$ for instance will resemble the dust distribution the same way as it resembles the gaussian shape of the dust cloud in Figure 2. Quantitative calculations of formation rates and molecular densities seem to be impossible at the moment for several reasons. One of them is that the chemical composition of the dust grains is still unknown although there is some evidence from recent results that silicates are the major constituents of interstellar dust clouds (Woolf, 1973). On these materials the reaction cross sections have to be measured at temperatures which are typical for interstellar grains. Calculating the number densities of interstellar molecules one also has to know the rates at which molecules are formed which do not have a permanent dipole momentum and thus can not be detected by radio spectroscopy. These molecules may consume a significant amount of the atoms and radicals which are available for reactions. 


\section{Acknowledgement}

This work was sponsored by the Deutsche Forschungsgemeinschaft, which gratefully is acknowledged.

\section{References}

Breuer, H. D.: 1969, Thesis, Bonn.

Hubbard, J. S., Hardy, J. P., and Horowitz, N. H.: 1971, Proc. Nat. Acad. Sci. 68, 574.

Moesta, H. and Breuer, H. D.: 1965, Rev. Sci. Instr. 36, 1372.

Moesta, H., Breuer, H. D., and Trappen, N.: 1969, Ber. Bunsenges. physik. Chemie 73, 879.

Solomon, P. M. and Klemperer, W.: 1973, preprint, to appear in Astrophys. J.

Stief, L. J.: 1971, Symposium on Interstellar Molecules, Charlottesville, to be published.

Woolf, N. J.: 1973, this volume, p. 485. 